\title{
NORMAL RADICALS AND NORMAL CLASSES OF MODULES
}

\author{
by W. K. NICHOLSON and J. F. WATTERS
}

(Received 6 May, 1986)

The study of special radicals was begun by Andrunakievic $[\mathbf{1}]$. A class $\mathscr{P}$ of prime rings is called special if it is hereditary and closed under prime extensions. The upper radicals determined by special classes are called special. In later works Andrunakievič and Rjabuhin [2] and [3] defined the concept of a special class of modules.

A left $R$-module $M$ is called prime if $R M \neq 0$ and every non-zero submodule has the same annihilator as $M$ (equivalently, if $I m=0$, where $I$ is an ideal of $R$ and $m \in M$, then either $m=0$ or $I M=0)$. Let $\mathscr{S}(R)$ be a class of prime $R$-modules and $\mathscr{S}=\bigcup \mathscr{S}(R)$, the union being over all rings $R$. Then $\mathscr{S}$ is called special if it satisfies the following conditions:

(S.1) for every ring $R, R$-module $M$, and ideal $I$ of $R$ with $I \subseteq(0: M), M \in \mathscr{S}(R)$ if and only if $M \in \mathscr{S}(R / I)$;

(S.2) if $M \in \mathscr{S}(R)$ and $I$ is an ideal of $R$ with $I M \neq 0$ then $M \in \mathscr{S}(I)$;

(S.3) if $I$ is an ideal of $R$ and $M \in \mathscr{S}(I)$ then $I M \in \mathscr{Y}(R)$.

If $\mathscr{S}$ is a special class of modules then

$$
\mathscr{P}=\{R: R \text { has a faithful module in } \mathscr{S}(R)\}
$$

is a special class of prime rings. Conversely, if $\mathscr{P}$ is a special class of rings and we set

$$
\mathscr{S}(R)=\left\{{ }_{R} M: M \text { is a prime } R \text {-module and } R /(0: M) \in \mathscr{P}\right\}
$$

then $\mathscr{S}=\bigcup \mathscr{S}(R)$ is a special class of modules.

The notion of a normal class of prime rings was defined in [5], where it was also shown that every such class is special and that a radical is normal and special if and only if it is the upper radical determined by a normal class. In this note we introduce the idea of a normal class of modules and prove that every normal class of prime rings is determined as above by a normal class of modules. It is also proved that such module classes are special and we note that the classes of prime modules, irreducible modules, and prime modules with non-zero socle are normal.

Normal classes of rings arise in studying rings connected in a Morita context. This is a four-tuple $\left[\begin{array}{cc}R & V \\ W & S\end{array}\right]$, where $R$ and $S$ are rings and ${ }_{R} V_{S}$ and ${ }_{S} W_{R}$ are bimodules, together with bimodule homomorphisms $V \otimes_{S} W \rightarrow{ }_{R} R_{R}, W \otimes_{R} V \rightarrow{ }_{S} S_{S}$ satisfying associativity conditions which are equivalent to insisting that $C=\left[\begin{array}{ll}R & V \\ W & S\end{array}\right]$ be an associative ring under the usual matrix operations. We shall refer to $C$ as the context ring. The context is called $S$-faithful if $S \neq 0$ and $V s W \neq 0$ for all non-zero $s \in S$. If $P$ is an ideal of $R$ then we denote $\{s \in S: V s W \subseteq P\}$ by $S_{P}$. 
Proposition 1 [5]. The following are equivalent for a class $\mathscr{P}$ of rings.

(a) If $\left[\begin{array}{ll}R & V \\ W & S\end{array}\right]$ is a Morita context and $P$ is an ideal of $R$ such that $R / P \in \mathscr{P}$ then either $S_{P}=S$ or $S / S_{P} \in \mathscr{P}$.

(b) If $\left[\begin{array}{ll}R & V \\ W & S\end{array}\right]$ is a Morita context and $R \in \mathscr{P}$ then either $S_{0}=S$ or $S / S_{0} \in \mathscr{P}$.

(c) If $\left[\begin{array}{ll}R & V \\ W & S\end{array}\right]$ is an $S$-faithful Morita context, then $R \in \mathscr{P}$ implies $S \in \mathscr{P}$.

A class $\mathscr{P}$ of prime rings is called normal if it satisfies the conditions of Proposition 1.

Definition 1. Let $\left[\begin{array}{ll}R & V \\ W & S\end{array}\right]$ be a Morita context. Then a context module is a pair of modules ${ }_{R} M,{ }_{S} N$ with module homomorphisms $\alpha: V \otimes_{S} N \rightarrow{ }_{R} M, \quad \beta: W \otimes_{R} M \rightarrow{ }_{S} N$ satisfying associativity conditions so that $D=\left[\begin{array}{l}M \\ N\end{array}\right]$ is a $C$-module for the context ring $C$ under the usual matrix operations.

Given a context $\left[\begin{array}{ll}R & V \\ W & S\end{array}\right]$ and an $R$-module $M$ we can construct an $S$-module $M^{\circ}$ so that $D=\left[\begin{array}{c}M \\ M^{\circ}\end{array}\right]$ is a context module. The construction appeared in [4].

For every $v \in V$ there is a $\mathbb{Z}$-morphism $v \cdot: W \otimes_{R} M \rightarrow M$ defined by $v \cdot(w \otimes m)=$ $(v w) m$ for all $w \in W, m \in M$. Put $X=\bigcap_{v \in V} \operatorname{ker}(v \cdot)$ and $M^{\circ}=(W \otimes M) / X$. Then $M^{\circ}$ is an $S$-module. The map $\beta$ of Definition 1 is given by $\beta: w \otimes m \rightarrow(w \otimes m)+X$ and we write this image as $w m$. The map $\alpha$ of Definition 1 is given by $\alpha: v \otimes n \rightarrow v \cdot t$, where $t \in W \otimes M$ and $n=t+X$. This image is written as $v n$. Thus $\left[\begin{array}{c}M \\ M^{\circ}\end{array}\right]$ is a $C$-module.

Some properties of this module are worth identifying here. A number of module properties are known to pass from $M$ to $M^{\circ}$ (see [4]). In particular if $M$ is faithful then $M^{\circ}$ is faithful. Also from the construction we have:

(a) if $n \in M^{\circ}$ and $V n=0$ then $n=0$;

(b) $M^{\circ}=W M$;

(c) if $M$ is faithful and $V S W \neq 0$ then $S M^{\circ} \neq 0$.

For (a), note that if $n=t+X, t \in W \otimes M$ then $v \cdot t=0$ for all $v \in V$; so $t \in X$ and $n=0$; (b) is clear from the definition of $M^{\circ}$ and $w m$, and (c) follows from (VS) $M^{\circ}=$ $(V S W) M$.

Definition 2. Let $\mathcal{N}(R)$ be a class of prime $R$-modules and $\mathcal{N}=\cup \mathcal{N}(R)$, the union being over all rings $R$. Then $\mathcal{N}$ is called normal if it satisfies (S.1) and

(N) for every context $\left[\begin{array}{ll}R & V \\ W & S\end{array}\right]$ and context module $D=\left[\begin{array}{l}M \\ N\end{array}\right]$ such that 
(i) for all $n \in N, V n=0$ implies $n=0$, and

(ii) $N=W M$ and $S N \neq 0, M \in \mathcal{N}(R)$ implies $N \in \mathcal{N}(S)$.

THEOREM. Let $\mathcal{N}$ be a normal class of modules. Then

$$
\mathscr{P}=\{R: R \text { has a faithful module in } \mathcal{N}(R)\}
$$

is a normal class of prime rings. Conversely, if $\mathscr{P}$ is a normal class of prime rings and we define, for every ring $R$,

$$
\mathcal{N}(R)=\left\{{ }_{R} M: M \text { is a prime } R \text {-module and } R /(0: M) \in \mathscr{P}\right\}
$$

then $\mathcal{N}=\cup \mathcal{N}(R)$ is a normal class of modules.

Proof. If $\mathcal{N}$ is a normal class of modules and $M \in \mathcal{N}(R)$ is a faithful $R$-module, then $(0: M)=0$ is a prime ideal of $R$ and $\mathscr{P}$, as defined, is a class of prime rings. From the comments after Definition 1, if $\left[\begin{array}{cc}R & V \\ W & S\end{array}\right]$ is an $S$-faithful Morita context then the context module $\left[\begin{array}{c}M \\ M^{\circ}\end{array}\right]$ satisfies (i) and (ii) of (N) and $M^{\circ}$ is faithful. Hence $M^{\circ} \in \mathcal{N}(S), S \in \mathscr{P}$, and $\mathscr{P}$ is a normal class of prime rings.

Now let $\mathscr{P}$ and $\mathcal{N}$ be as in the statement of the converse. For (S.1), suppose that $M$ is an $R$-module and $I$ is an ideal of $R$ with $I \subseteq(0: M)$. Put $\bar{R}=R / I$. Note that $(0: M)_{\bar{R}}=(0: M) / I$ and if $r \in R$ and $\bar{r}=r+I$ then $\bar{r} m=r m$ for all $m \in M$. Thus $\bar{R} /(0: M)_{\bar{R}} \cong R /(0: M)$ and $M$ is a prime $R$-module if and only if it is a prime $\bar{R}$-module. Therefore $M \in \mathcal{N}(R)$ if and only if $M \in \mathcal{N}(\bar{R})$.

For (N), suppose that the context and context module are as described in Definition 2 and that $M \in \mathcal{N}(R)$. To see that $N$ is a prime $S$-module, let $J$ be an ideal of $S$ and $n \in N$ with $J n=0$. Then $(V J W)(V n)=0$; so either $(V J W) M=0$ or $V n=0$, since $M$ is a prime $R$-module. If $V n=0$ then $n=0$ from (i). If $(V J W) M=0$ then $V J N=0$ from (ii) and $J N=0$ from (i). Thus $N$ is a prime $S$-module. To prove that $S /(0: N) \in \mathscr{P}$, observe that $(0: N)=\{s \in S: V s W \subseteq(0: M)\}$ from (i) and (ii) in (N). From Proposition 1 (a), either $(0: N)=S$ or $S /(0: N) \in \mathscr{P}$. Since $S N \neq 0$, by hypothesis, it follows that $S /(0: N) \in \mathscr{P}$.

Proposition 2. Every normal class of modules is special.

Proof. Let $\mathcal{N}$ be a normal class of modules. Let $M \in \mathcal{N}(R)$ and $I$ be an ideal of $R$ with $I M \neq 0$. Consider the context $\left[\begin{array}{cc}R & I \\ R^{1} & I\end{array}\right]$ and context module $D=\left[\begin{array}{l}M \\ M\end{array}\right]$. If $I m=0$, $m \in M$, then $m=0$ since $M$ is a prime $R$-module and $I M \neq 0$. Therefore the conditions (N) (i) and (N) (ii) from Definition 2 are satisfied and so $M \in \mathcal{N}(I)$. This establishes (S.2). For (S.3), let $I$ be an ideal of a ring $R$ and $M \in \mathcal{N}(I)$. Consider the context $\left[\begin{array}{cc}I & R^{1} \\ I & R\end{array}\right]$ and context module $\left[\begin{array}{c}M \\ I M\end{array}\right]$. Since $M$ is a prime $I$-module, $I M \neq 0$ and 
$I(I M) \neq 0$. Hence $R(I M) \neq 0$ and the conditions (N) (i) and (N) (ii) of Definition 2 are satisfied; so that $I M \in \mathcal{N}(R)$.

EXAmples. The three classes we shall consider here were shown to be special in [3]. Thus (S.1) is satisfied. We shall use the notation of $(\mathrm{N})$.

1. The class of all prime modules is normal. Let $M$ be a prime $R$-module, $J$ an ideal of $S$, and $n \in N$. As in the proof of the Theorem, $J n=0$ implies $J N=0$ or $n=0$; so $N$ is a prime $S$-module.

2. The class of all irreducible modules is normal. Let $M$ be an irreducible $R$-module and $n \in N, n \neq 0$. Then $0 \neq V n$; so $V n=M$ and $S n \supset W V n=W M=N$. Thus $N$ is an irreducible $S$-module.

3. The class of all prime modules with non-zero socle is normal. Let $M$ be a prime $R$-module with minimal submodule $K$. If $(V W) K=0$ then $(V W) M=V N=0$, which implies that $N=0$. But $S N \neq 0$; so $(V W) K \neq 0$. Hence $W K \neq 0$. Let $n \neq 0, n \in W K$. Then $0 \neq V n \subseteq K$ and, by the minimality of $K, V n=K$. Therefore $S n \supseteq(W V) n=W K$ and $W K$ is a minimal submodule of $N$. Along with Example 1 this proves the normality of this class.

Remark. It was shown in [3] that the class $\mathscr{P}$ determined by the module classes in Examples 2 and 3 is the class of primitive rings.

\section{REFERENCES}

1. V. A. Andrunakievič, Radicals of associative rings. II. Examples of special radicals, Mat. Sb. (N.S.) 55 (97) (1961), 329-346; Amer. Math. Soc. Transl. (2) 52 (1966), 129-150.

2. V. A. Andrunakievic and Ju. M. Rjabuhin, Special modules and special radicals, Dokl. Akad. Nauk SSSR 147 (1962), 1274-1277.

3. V. A. Andrunakievič and Ju. M. Rjabuhin, Special modules and special radicals, In Memoriam: N. G. Čebotarev, 7-17, Izdat. Kazan. Univ., Kazan, 1964.

4. G. Desale and W. K. Nicholson, Endoprimitive rings, J. Algebra 70 (1981), 548-560.

5. W. K. Nicholson and J. F. Watters, Normal radicals and normal classes of rings, J. Algebra 59 (1979), 5-15.

Department of Mathematics and Statistics

The University of CaLgary

Calgary

AlberTa

CANADA T2N 1N4
Department of Mathematics THE UNIVERSITY OF LEICESTER

LEICESTER

ENGLAND LE1 7RH 